\title{
LES GARDES - FRONTIÈRES AU TRAVAIL. LA CONSTRUCTION DE LA FRONTIÈRE EUROPÉENNE
}

Chantiers de recherche

Sara Casella-Colombeau

\section{L'Harmattan | Politique européenne}

\author{
$2009 / 1-n^{\circ} 27$ \\ pages 233 à 240
}

ISSN 1623-6297

Article disponible en ligne à l'adresse:

http://www.cairn.info/revue-politique-europeenne-2009-1-page-233.htm

Pour citer cet article :

Casella-Colombeau Sara, «Les gardes - frontières au travail. La construction de la frontière européenne 》 Chantiers de recherche,

Politique européenne, 2009/1 n²7, p. 233-240.

Distribution électronique Cairn.info pour L'Harmattan.

(c) L'Harmattan. Tous droits réservés pour tous pays.

La reproduction ou représentation de cet article, notamment par photocopie, n'est autorisée que dans les limites des conditions générales d'utilisation du site ou, le cas échéant, des conditions générales de la licence souscrite par votre établissement. Toute autre reproduction ou représentation, en tout ou partie, sous quelque forme et de quelque manière que ce soit, est interdite sauf accord préalable et écrit de l'éditeur, en dehors des cas prévus par la législation en vigueur en France. II est précisé que son stockage dans une base de données est également interdit. 


\section{CHANTIERS DE RECHERCHE}

\section{Sara Casella-Colombeau}

\section{LES GARDES-FRONTIÈRES AU TRAVAIL. LA CONSTRUCTION DE LA FRONTIÈRE EUROPÉENNE}

Le but de cette recherche est d'analyser dans une perspective comparative les conséquences des politiques européennes de contrôle des frontières extérieures sur les services administratifs responsables de la surveillance des frontières européennes en France, en Espagne et en Italie. Il est en effet intéressant de constater que les évolutions récentes des politiques européennes de gestion des frontières extérieures mettent en jeu une redéfinition des frontières nationales en tant que lieu d'exercice souverain des représentants de l'État et participent ainsi, dans le cadre de la construction d'une frontière européenne unique, à la transformation du rapport entre États et communauté politique nationale et européenne.

Létude comparative du groupe professionnel des gardes-frontières, peu étudié jusqu'alors, conduit dès lors à s'interroger sur les possibilités et les modalités de la création d'un Corps européen de gardes-frontières identifié à sa mission plus qu'aux appartenances nationales.

\section{Cadre européen}

Deux phénomènes ont conduit les États-membres à élaborer une politique de gestion des frontières au niveau européen : d'une part, la volonté de compenser la liberté de circulation interne à l'espace Schengen, renforcée par le processus d'élargissement dans lequel s'engage alors l'Union européenne. D'autre part, l'insistance de la part des États de "première frontière $"^{1}$ (notamment des États du Sud de l'Union Européenne) à partager le « fardeau » de la surveillance des frontières.

\footnotetext{
1 Dont les frontières externes correspondent à des frontières extérieures de l'Union européenne.
}

politique européenne, $\mathrm{n}^{\circ} 27$, hiver 2009, p. 233-240. 
Depuis la signature du traité de Maastricht en 1992 et la création du " troisième pilier ", la politique de contrôle et de surveillance des frontières a peu à peu été intégrée dans le domaine communautaire.

Ce processus s'est accompagné d'un débat sur les modalités d'organisation des gardes-frontières européens et notamment sur la création d'un Corps européen de gardes-frontières. Au début des années 2000, les États-membres formulent le souhait de voir la coopération en matière de contrôle et de surveillance des frontières s'institutionnaliser. En octobre 2004, le Conseil adopte un règlement portant création de Frontex, l'agence européenne de coordination de la coopération opérationnelle pour la gestion des frontières extérieures.

Cette structure communautaire est le fruit d'un compromis entre les tenants de la création d'un Corps européen de gardes-frontières et les États-membres soucieux de préserver leur souveraineté nationale. Frontex assure ainsi la définition d'un cadre d'action commun à tous les gardes-frontières européens, notamment par la coordination d'opérations menées par différents corps de gardes-frontières nationaux à des points précis de la frontière européenne. Mais l'agence ne disposant d'aucun moyen propre, elle dépend des États-membres pour la mise en œuvre des missions qu'elle organise.

Parallèlement, les relations avec les pays voisins du Sud, notamment du bassin méditerranéen ont progressivement intégré la question de la gestion des frontières. Les accords concernant le contrôle de l'émigration par les États-tiers et l'organisation de patrouilles communes dans le cadre de l'agence Frontex avec les gardes-frontières mauritaniens, sénégalais et marocains sont autant de conséquences pratiques de la construction de cet " espace commun de paix, de stabilité et de prospérité $»^{2}$.

\section{Dimension théorique}

Il s'agira, dans cette recherche de s'intéresser principalement aux dynamiques de transformation des politiques publiques, qu'elles soient définies au niveau européen ou national. Ce cadrage théorique et méthodologique conduit à considérer trois dimensions théoriques.

La première dimension est celle relative à la construction de l'Union européenne. Les politiques de gestion des frontières extérieures de

2 Déclaration de Barcelone, http://europa.eu/scadplus/leg/fr/lvb/r15001.htm 
l'Union européenne ont été principalement abordées sous l'angle du processus de l'intégration européenne et de la mise en place au niveau européen de projets concrets et de réseaux d'acteurs favorisant le transfert au niveau européen des responsabilités qui incombaient jusqu’à présent aux États régaliens (Guiraudon, 2000, 2001, 2003 ; Monar, 2001, 2004). Ces études mettent l'accent sur les «law and order officials responsible for migration control", fonctionnaires " européanisés " des ministères de l'Intérieur des pays membres, qui, au moment de la construction du domaine Justice, Liberté, Sécurité (anciennement Justice et Affaires intérieures) ont acquis une place fondamentale dans le processus de décision visant à la définition des politiques européennes.

L'examen des conséquences des politiques européennes nous conduit à nous intéresser d'avantage au processus d'européanisation qu'au processus d'intégration.

Cependant, les études sur l'européanisation s'intéressent le plus souvent aux relations entre acteurs politico-administratifs au plus haut niveau, laissant de côté la question de la mise en œuvre et de l'effectivité véritable de ces politiques au niveau local ou micro. Ainsi, Maria Green Cowles, James Caporaso et Thomas Risse $(2001)^{3}$ décrivent l'européanisation comme un processus principalement "top-down", qui conduit à des changements dans les "structures nationales " par l'intermédiaire de pressions adaptatives qui s'exercent selon la "goodness of fit " des institutions nationales par rapport aux politiques communautaires. Ces pressions s'appliquent de manière différenciée selon les facteurs de médiation propres à chaque "structure nationale ". Ces auteurs se concentrent donc sur des facteurs institutionnels et leur question principale reste celle de la présence ou non d'un changement dû à ces pressions adaptatives sur les "structures nationales". Ils ne s'intéressent pas aux acteurs de la mise en ouvre des politiques communautaires.

Olivier Nay $(2001)^{4}$ prend en compte les agents de la mise en ouvre des programmes européens mis en place dans le cadre de la politique

\footnotetext{
3 Maria Green Cowles, James Caporaso et Thomas Risse,Transforming Europe: Europeanization and Domestic Change, New York, Cornell University Press, 2001.

4 Olivier Nay, " Négocier le partenariat. Jeux et conflits dans la mise en œuvre de la politique communautaire en France ", Revue française de science politique, vol. 51, n 3 , juin, 2001, p. 459-481.
} 
des fonds structurels Il montre que ces programmes sont l'occasion d'une reconfiguration des relations de pouvoir entre les services déconcentrés de l'État et les responsables politiques régionaux menant à une mise en œuvre sensiblement différente selon les régions. Il s'intéresse donc aux "décideurs", aux acteurs pour qui la mise en œuvre des programmes européens est l'occasion de remettre en jeu l'équilibre des forces au sein des élites politiques et administratives régionales.

Notre projet vise au contraire à porter notre attention sur « l'européanisation par le bas ", à questionner le processus d'européanisation dans ces effets sur les pratiques des acteurs de terrain, dans notre cas, les gardes-frontières.

Nous voulons insister dans notre recherche sur l'importance de l'interprétation de la règle et du pouvoir discrétionnaire ("discretion») des "street-level bureaucrats" 5 . Nous nous appuyons pour cela sur le travail d'Alexis Spire (2005), qui s'est intéressé au travail de création normative des fonctionnaires de préfecture chargés de la délivrance des titres de séjour et de travail.

A. Spire, prenant en compte ces " normes secondaires d'application " montre l'importance du processus de création normative dans le travail des agents intermédiaires des préfectures. Il met en évidence un "ethos préfectoral ", c'est-à-dire l'« ensemble des principes et des dispositions à agir qui organisent les pratiques des agents de préfecture $"^{6}$. Cet ethos permet de réguler la création normative ayant lieu à chaque niveau de la mise en ouvre. C'est ce qui permet à un ensemble de décisions prises par des acteurs à la rationalité limitée de tenir ensemble. La mobilisation de cet auteur nous permet de nous interroger sur la présence d'un ethos propre aux gardes-frontières d'un État, ou d'une région.

La littérature sur la mise en ouvre des politiques européennes nous fournit un exemple proche de l'" ethos " décrit par A. Spire, les " world of compliance» du modèle de Gerda Falkner ${ }^{7}$. G. Falkner s'intéresse à la manière dont les acteurs politiques et administratifs de quinze États-

${ }^{5}$ Michael Lipsky, Street-level Bureaucracy, New York, Russel Sage Foundation, 1980.

6 Alexis Spire, Etrangers à la carte : l'administration de l'immigration en France, 19451975, Paris, Grasset, 2005, p. 169

7 Gerda Falkner, Miriam Hartlapp et Olivier, Treib, «Worlds of Compliance: Why Leading Approaches to European Union Implementation are only "sometimes-true theories' », European Journal of Political Research, vol. 46, n 3, 2007, p. 416. 
membres effectuent la transposition de directives européennes dans leur droit national. Elle définit ainsi une typologie composée de trois " world of compliance " qui permet d'expliquer et de prévoir les caractéristiques des transpositions des directives européennes dans ces différents pays. De la même manière nous pouvons envisager d'identifier des idéauxtype de la manière dont les acteurs de terrain réinterprètent les règles définies au niveau européen.

Nous pourrions identifier des "world of compliance » de la mise en œuvre des politiques européennes de contrôle des frontières au niveau local, déterminé par des pratiques professionnelles plus que par des appartenances nationales.

La deuxième dimension est liée à l'identification des différentes phases de l'action publique et à l'attention portée sur l'une d'elles, la phase de mise en œuvre des politiques publiques. À l'instar de Pierre Lascoumes ${ }^{8}$ nous considérons que « la mise en ouvre d'une politique publique, sa dynamique de diffusion et d'implantation peuvent être [...] envisagées sous l'angle d'un processus continu de création normative secondaire. ». La production de normes résultant du travail concret des acteurs transforme les règles élaborées au niveau central. Cette hypothèse nous conduit à nous interroger sur la présence " d'effets retour " liés à la création de normes et l'interprétation par les acteurs de la mise en œuvre de ces politiques sur les décisions des autorités européennes.

Enfin, la troisième dimension reflète un intérêt pour les pratiques des acteurs en ce qu'elles révèlent à la fois leur identité et renseignent sur les structures organisationnelles de l'administration, il s'agit d'une sociologie des acteurs de terrain et de l'administration. Nous mobiliserons également les travaux spécifiques à l'étude des forces de l'ordre.

Cette recherche conduit à considérer à la fois les travaux mettant en avant le processus de création normative dans le travail des agents de terrain mais aussi des études sur l'européanisation de l'administration, sur les évolutions de la structuration de l'administration?.

\footnotetext{
8 Pierre Lascoumes, "Normes et mise en œuvre des politiques publiques ", L'Année sociologique, vol. 40, 1990, p. 62.

9 Johan Olsen, "Towards a European Administrative Space? ", Journal of European Public Policy, vol. 10, n ${ }^{\circ}$, 2003, p. 506-531.
} 


\section{Aspects prospectifs et méthodologiques}

La construction du cadre comparatif de ma recherche traduit une interrogation sur l'origine de l'homogénéisation supposée des pratiques des gardes-frontières dans l'Union européenne. Il repose sur deux séries d'hypothèses. La première porte sur les spécificités du niveau national dans la mise en œuvre des politiques européennes. La deuxième série d'hypothèses permet de mettre en lumière le rôle des pratiques professionnelles dans ces mêmes politiques publiques.

La première hypothèse met en effet l'accent sur la prise en compte des spécificités nationales dans l'étude des politiques européennes. Elle postule en particulier que l'influence des politiques européennes sur les politiques nationales de ces États a été d'autant plus forte qu'elles n'avaient pas de spécificités avant la communautarisation des questions de gestion des frontières. Cette hypothèse permet de définir les termes de l'analyse comparative.

Les pays du Sud de l'Europe ont vu leur solde migratoire s'inverser au cours des dernières décennies. L'Espagne n'est devenue un pays d'immigration qu'en 2001. En 2000, ce pays comptait 923879 citoyens résidants d'autres nationalités et 4002509 en 2006. En Italie on recensait 1270553 résidants étrangers en 2000, il y en avait 2670514 en $2006^{10}$.

Au-delà des chiffres, la pression qui s'exerce sur les gouvernants en faveur d'une redéfinition des politiques de gestion des frontières extérieures est également liée au traitement médiatique de l'arrivée sur leurs côtes des embarcations de fortune utilisées par les migrants en provenance des côtes africaines. L'immigration est devenue un thème central des débats politiques nationaux de ces États.

Par ailleurs, l'Espagne et l'Italie ont une histoire vis-à-vis de leurs politiques migratoires relativement semblable, liée notamment à la mise en place tardive d'une législation sur l'immigration. Ce n'est que sous l'influence européenne et après la signature des accords de Schengen que ces États établissent des règles strictes concernant le franchissement de leurs frontières. Si, dans un premier temps, la législation fut jugée permissive par les autres pays de l'Union européenne, on assiste par la

10 Source eurostat, http://epp.eurostat.ec.europa.eu/portal/page?_pageid=1996, $45323734 \&$ dad=portal\&_schema=PORTAL\&screen=welcomeref\&open=/C/C6\&1 anguage $=$ fr\&product $=$ Yearlies_new_population\&root=Yearlies_new_population\&scr ollto $=0$ 
suite à un durcissement des conditions d'entrée, de séjour et de régularisation des migrants. Le risque migratoire élevé à leurs frontières maritimes et leurs politiques de régularisation sont autant d'éléments qui les placent dans un Most Similar System Design ${ }^{11}$, qui correspond à la volonté de " comparer des systèmes politiques qui partagent des caractéristiques similaires pour essayer de neutraliser certaines différences afin d'en mettre d'autres en évidence ${ }^{12}$.

La France jouera le rôle du cas de contrôle. En effet, la France est un ancien pays d'immigration dont l'accès au séjour régulier sur des critères économiques a été limité à partir du milieu des années 1970 . Pourtant, les flux se sont maintenus et la régularisation des immigrés peut avoir lieu dans certains cas grâce au regroupement familial et à la reconnaissance du droit d'asile. Les frontières maritimes de la France ne sont pas des points d'entrée important des migrants.

La deuxième hypothèse porte sur les pratiques, en tant que fonctionnaires de terrain, des policiers de la PAF (Police aux frontières) français, des militaires de la Guardia Civile espagnole, ou de la Guardia di Finanza et des Carabinieri italiens. Une des hypothèses fortes de notre thèse est que la variable fondamentale dans l'étude de la mise en œuvre des politiques de contrôle des frontières extérieures de l'Union européenne est la " discretion "13 des gardes-frontières. La littérature spécialisée dans l'étude des "street-level bureaucrats " ${ }^{14}$ définit ainsi le libre arbitre dont disposent ces agents, la capacité de sélectionner ou d'interpréter les règles définies par leur hiérarchie.

En effet, la Guardia Civile comme les Carabinieri ou la Guardia di Finanza ont des traditions de corps militaires et des pratiques professionnelles qui préexistent aux politiques européennes.

Nous faisons l'hypothèse que la diversité des formes d'interprétation d'une norme européenne unique s'explique par des variables relatives à l'organisation administrative et à la spécificité des corps de gardesfrontières (le type de frontière contrôlée ou surveillée - maritime ou aérienne, nationale ou européenne - ; le type d'activité principale du gardefrontière ; son grade ; le type de corps auquel il appartient - militaire ou civil -).

11 Todd Landman, Issues and Methods in Comparative Politics an Introduction, Londres, Routledge, 2003 [2e ed.], p. 29.

12 Ibid.

13 Michael Lipsky, op. cit.

14 Ibid. 
La deuxième hypothèse détermine les méthodes d'enquêtes de cette recherche. L'enquête de terrain consistera en des entretiens avec des responsables nationaux ainsi qu'avec des responsables et des fonctionnaires de terrain locaux des services de contrôle aux frontières dans ces trois pays. L'observation non participante est également prévue.

Plusieurs points de la frontière seront étudiés pour chaque pays afin d'avoir accès à des informations concernant le travail des gardesfrontières aux frontières aériennes et maritimes dans différentes régions.

Les terrains envisagés pour l'Italie et l'Espagne, à savoir l'île de Lampedusa et les îles Canaries, sont relativement semblables : situées entre le territoire européen et les côtes africaines elles sont devenues en effet des points de passage privilégiés des migrants tentant de pénétrer de façon irrégulière dans l'espace Schengen.

D'autres points d'observation à la frontière sont aussi envisagés comme l'aéroport de Madrid et la côte andalouse où on assiste cette année à une recrudescence des arrivées suite à l'efficacité des contrôles autour des îles Canaries. En Italie, les côtes du Sud-Est de la Sicile et l'aéroport de Rome sont aussi envisagés.

En France, l'aéroport de Roissy est un des lieux d'arrivée majeurs des migrants pénétrant sur le territoire français. 59,9 millions de voyageurs transitent chaque année par cet aéroport qui a été classé en deuxième position en Europe en termes de passagers en 2007. En 2006, 16397 personnes ont été placées en zone d'attente, lieu de maintien des migrants non admis sur le territoire à leur passage du poste frontière ${ }^{15}$. Il semble donc que les contrôles effectués dans cet aéroport soient un enjeu majeur dans le contrôle migratoire français. D'autre part, même si le flux de migrants y est beaucoup moins important, un grand port est envisagé comme terrain potentiel, Marseille ou Bordeaux par exemple.

Le but de cette recherche est d'identifier les dynamiques à l'œuvre dans la transformation des pratiques des fonctionnaires de terrain dans le cadre de la construction d'une frontière européenne. Cette identification nous permettra dans un deuxième temps de définir la nature du Corps européen de gardes-frontières en formation.

\footnotetext{
15 Ce chiffre ne prend pas en compte les migrants renvoyés dans la même journée et qui n'ont de ce fait pas été placés dans ce centre.
} 